\title{
Estado nutricional e consumo alimentar de adolescentes de um centro de juventude da cidade de São Paulo ${ }^{1}$
}

\section{Nutritional status and food consumption of adolescents registered at a center of youth from the city of São Paulo, Brazil}

Giorgia Christina Barbosa GARCIA

Ana Maria Dianezi GAMBARDELLA ${ }^{3}$

Maria Fernanda Petrole FRUTUOSO ${ }^{4}$

\section{RE S U M O}

Foram avaliados o estado nutricional e o consumo alimentar de 153 adolescentes dos sexos masculino e feminino, com média de idade de 11,90 $\pm 1,26$ anos, de baixo nível socioeconômico, matriculados em um Centro de Juventude da cidade de São Paulo. Para a classificação do estado nutricional foram utilizados como pontos de corte os percentis da distribuição de Índice de Massa Corporal por idade e sexo. Aplicou-se recordatório de 24 horas, com auxílio do álbum de registros fotográficos, para estimar o consumo alimentar. Calculou-se o Índice de Qualidade Nutricional para cálcio e ferro. As médias dos valores de energia, proteína, carboidrato, lipídios, cálcio, ferro e colesterol foram de $1953 \mathrm{kcal}, 69 \mathrm{~g}, 264 \mathrm{~g}, 69 \mathrm{~g}, 517 \mathrm{mg}, 10 \mathrm{mg}$ e $329 \mathrm{mg}$, respectivamente. Com relação ao estado nutricional, $78,4 \%$ dos adolescentes encontravam-se na faixa de normalidade, $11,8 \%$ apresentavam risco de sobrepeso, 7,8\% estavam com sobrepeso e $2,0 \%$ tinham baixo peso. Detectou-se consumo insuficiente de cálcio, especialmente entre as meninas $(98,4 \%)$, que também apresentaram maior proporção de qualidade da dieta "pobre" e "regular" em ferro (73,8\%).

Termos de indexação: adolescentes, estado nutricional, consumo de alimentos, cálcio.

\section{A B S T R A C T}

This paper evaluated the nutritional status and the food consumption of 153 adolescents (males and females), with a median age of 11.9 years $( \pm 1.26)$, and with low socioeconomic level, registered at a Center of Youth

\footnotetext{
1 Pesquisa financiada pela Fundação de Auxílio à Pesquisa de São Paulo (FAPESP). Processo no 99/07621-1.

2 Mestranda em Nutrição, Faculdade de Saúde Pública, Universidade de São Paulo. Bolsista da FAPESP, (processo $n^{\circ}$ 99/07621-1)

3 Departamento de Nutrição, Faculdade de Saúde Pública, Universidade de São Paulo. Av. Dr. Arnaldo, 715, 01246-904, São

Paulo, SP, Brasil. Correspondência para/Correspondence to: A.M.D. GAMBARDELLA. E-mail: gambarde@usp.br

${ }^{4}$ Faculdade de Saúde Pública, Universidade de São Paulo.
} 
from the city of São Paulo. To evaluate the nutritional status, cutoff points based on percentiles of the distribution of Body Mass Index for age and sex were adopted. A 24-hour recall, with an album of photographic registers, was applied to estimate the food consumption. The Nutritional Quality Index for calcium and iron was calculated. The average values of energy, protein, carbohydrate, lipids, calcium, iron and cholesterol were 1953kcal, 69g, 264g, 69g, 517mg, 10mg and 329mg, respectively. According to the assessment of the nutritional status, 78.4\% of the adolescents were within the range of normality, $11.8 \%$ presented overweight risk, $7.8 \%$ were overweight and $2.0 \%$ had low weight. Insufficient calcium consumption was detected, especially among girls (98.4\%), who had also presented unsatisfactory quality of diet in iron (73.8\%).

Index terms: adolescents, nutritional status, food consumption, calcium.

\section{N T R O D U Ç Ã O}

A adolescência consiste no período de transição entre a infância e a vida adulta, caracterizado por intensas mudanças somáticas, psicológicas e sociais, compreendendo a faixa etária dos 10 aos 19 anos de idade. Na fase inicial da adolescência (10 a 14 anos), chamada de puberdade, ocorrem o estirão de crescimento, o aumento rápido das secreções de diversos hormônios e o aparecimento dos caracteres sexuais secundários (maturação sexual). A fase final (15 a 19 anos) caracteriza-se pela desaceleração destes processos (Organización Mundial..., 1995).

Vários fatores interferem no consumo alimentar neste período da vida, tais como valores socioculturais, imagem corporal, convivências sociais, situação financeira familiar, alimentos consumidos fora de casa, aumento do consumo de alimentos semipreparados, influência exercida pela mídia, hábitos alimentares, disponibilidade de alimentos, facilidade de preparo (Dietz, 1998).

Aparentemente, a dieta de adolescentes caracteriza-se pela preferência por produtos alimentícios com inadequado valor nutricional, ou seja, aqueles com elevado teor de gordura saturada e colesterol, além de grande quantidade de sal e açúcar (Dietz, 1998). O consumo excessivo desses alimentos pode provocar aumento na quantidade de gordura corporal e, em decorrência, sobrepeso. Estudos epidemiológicos demonstram que sobrepeso em adolescentes está associado à elevação do risco de sobrepeso na vida adulta e, conseqüentemente, ao aumento do risco de doenças coronarianas e degenerativas, além de maior incidência de câncer de cólon e reto (Andersen et al., 1995; Dietz 1998 ).

Há evidências de que a prevalência de sobrepeso e obesidade vem aumentando no mundo todo. A tendência secular de aumento da incidência de obesidade foi registrada na América (Brasil, Estados Unidos e Canadá), Europa (Inglaterra, Finlândia, Alemanha, Holanda e Suécia, entre outros países) e região ocidental do Pacífico (Austrália e Samoa) (World Health..., 1997).

A importância de se conhecer o consumo alimentar prende-se ao fato de existir correlação positiva entre dieta e risco de morbimortalidade. Dietas inadequadas, aquelas com elevado teor de lipídios, energia e carboidratos simples, podem ser consideradas fator de risco para doenças crônicas e obesidade (Andersen et al., 1995). Assim, destaca-se a relevância de estudos que verifiquem a prevalência de sobrepeso e obesidade, principalmente entre adolescentes, e, ainda, a relação entre inadequação de dietas e risco para doenças crônicas e obesidade.

Diante do exposto, este trabalho visa avaliar o estado nutricional e o consumo alimentar de adolescentes dos sexos masculino e feminino, matriculados em um Centro de Juventude da cidade de São Paulo.

\section{CASUÍSTICA E MÉTODOS}

Este estudo foi parte do projeto 'Consumo alimentar, atividade física e prevalência de 
sobrepeso, obesidade, hipertensão arterial em adolescentes e seus pais', desenvolvido no Centro de Juventude da Achiropita, situado na região central do município de São Paulo.

Centros de Juventude (CJ) são entidades assistenciais, sem fins lucrativos, conveniados com a Prefeitura, que realizam trabalhos de auxílio à comunidade carente (renda inferior a quatro salários mínimos), atendendo, gratuitamente, indivíduos de 7 a 14 anos de idade, em regime de 4 horas/dia, de segunda a sexta-feira, sendo pré-requisito freqüentar escola no período oposto ao do CJ. Esses Centros oferecem duas refeições por período (desjejum e almoço ou almoço e lanche da tarde), as quais devem suprir $50 \%$ das necessidades energéticas e protéicas diárias. Além disso, desenvolvem-se programas de atendimento socioeducativo, como complementação escolar, iniciação desportiva, recreação, lazer e cultura. Para a faixa de 12 a 14 anos estão previstas atividades voltadas ao fortalecimento da auto-estima, com ênfase no sucesso escolar e em atividades de sensibilização para o trabalho (São Paulo..., 1993).

O CJ selecionado para o desenvolvimento do projeto mantinha convênio com a Prefeitura de São Paulo e recebia doações da comunidade provenientes de um montante arrecado anualmente em uma tradicional festa italiana da cidade.

$O$ estudo foi realizado com todos os adolescentes de 10 a 14 anos de idade matriculados no CJ, perfazendo um total de 153 adolescentes.

A coleta de dados foi realizada de setembro a novembro de 1999. A direção do Centro forneceu listagem com os nomes, sexo e data de nascimento de todos os alunos, separados por turmas. As entrevistas individuais, conduzidas por pesquisadores treinados, ocorreram no próprio Centro. Foram aplicados um questionário contendo questões sobre informações sociais e atividades físicas e um inquérito alimentar utilizando o método de recordatório de 24 horas, e foram mensurados o peso e a estatura corporais.
A idade foi calculada em anos completos, mediante a diferença entre a data de nascimento e a data da entrevista.

Para a medida do peso corporal utilizou-se balança eletrônica do tipo plataforma com capacidade para $150 \mathrm{~kg}$ e graduação em $100 \mathrm{~g}$ (Tanita TFB-521 ${ }^{\circledR}$ ), e os adolescentes trajaram roupas leves e ficaram descalços (Gordon et al., 1988).

A estatura foi mensurada através de estadiômetro $\left(\mathrm{Seca}^{\circledR}\right)$ fixado à parede com escala em milímetros $(\mathrm{mm})$, conforme metodologia proposta por Gordon et al. (1988), sendo realizadas duas medidas e considerada a média dos valores obtidos.

Procurou-se identificar as atividades físicas praticadas pelos adolescentes segundo o tipo e a duração (em horas por dia e em dias por semana), bem como o tempo destinado aos estudos, lazer e descanso. A atividade física foi classificada conforme o dispêndio energético em leve, moderada e intensa (Organização Mundial..., 1998).

Para a avaliação do estado nutricional calculou-se o Índice de Massa Corporal (IMC), adotando como critério de classificação os valores para idade e sexo e os respectivos pontos de corte propostos pela Organização Mundial de Saúde (OMS) (World Health..., 1995).

Visando facilitar a entrevista sobre o consumo alimentar, utilizou-se o método de recordatório de 24 horas de um dia juntamente com álbum de registros fotográficos (Zabotto et al., 1996). Tal procedimento foi necessário, pois, durante o pré-teste realizado com adolescentes da mesma faixa etária, notou-se que eles não apresentavam dificuldades em lembrar dos alimentos ingeridos no dia anterior, porém consideravam difícil determinar a quantidade. As entrevistas eram realizadas de segunda a sexta e os adolescentes relatavam o que consumiam no dia anterior.

O método recordatório de 24 horas consiste no relato de todos os alimentos 
consumidos no período de 24 horas, desde a primeira até a última refeição realizada neste intervalo de tempo. Tem sido freqüentemente utilizado em estudos de epidemiologia analítica sobre saúde, doença e consumo alimentar, pois permite uma estimativa mais exata do consumo médio diário e constitui um procedimento que não demanda muito tempo para sua aplicação (Majem \& Barba, 1995).

Os alimentos e/ou preparações informados pelos entrevistados foram convertidos em medidas caseiras e posteriormente em peso (gramas), conforme elucidado no registro fotográfico (Zabotto et al., 1996). As quantidades dos alimentos e/ou preparações não contempladas em fotos foram estimadas de acordo com relatos, procurando-se identificar o tipo de preparação baseado no modo como era preparado o alimento e nos ingredientes contidos.

Os cálculos para quantificar o valor energético total da dieta, em macronutrientes (proteína, carboidrato e lipídio), micronutrientes (ferro e cálcio) e colesterol, foram realizados com auxilio do Software Diet Win (Reinstem, 1998).

A necessidade energética de cada adolescente foi calculada pelo método preconizado pela Organización Mundial... (1998), que utiliza o dispêndio de metabolismo basal e a atividade física.

Para a análise da adequação do consumo de energia, proteína, cálcio e ferro foram adotados como padrão valores entre $80 \%-120 \%$ do recomendado para a população norte-americana, e adotou-se valor equivalente a $100 \%$ do preconizado pelo National Research Council (National Research...,1989) para o colesterol.

Foi calculado o Índice de Qualidade Nutricional (IQN) para cálcio e ferro, conforme critério proposto por Wittwer et al. (1977).

Os dados foram analisados com auxílio do programa estatístico Statistical Package for the Social Sciences (SPSS), segundo medidas de tendência central e de dispersão (Statistical..., 1996). Foi calculado o Qui-Quadrado para verificar a relação entre o estado nutricional e os parâmetros da prática esportiva, sexo e idade. Calculou-se também o teste não paramétrico Kruskal-Wallis para avaliar a relação entre sexo e consumo alimentar.

\section{RESULTADOS}

As médias e desvios-padrão de peso, altura e IMC para o sexo masculino foram $44,0 \mathrm{~kg} \pm 12,71,150,3 \mathrm{~cm} \pm 10,38 \mathrm{e}$ $19,18 \mathrm{~kg} \pm 3,59 \mathrm{~kg} / \mathrm{m}^{2}$ e para o sexo feminino foram $45,5 \mathrm{~kg} \pm 10,36,151,4 \mathrm{~cm} \pm 8,94 \mathrm{e}$ $19,22 \mathrm{~kg} / \mathrm{m}^{2} \pm 3,17$, respectivamente, sem diferenças estatisticamente significativas entre os sexos.

Com relação ao estado nutricional, verificou-se que $2,2 \%$ dos meninos e $1,6 \%$ das meninas encontravam-se abaixo do peso ideal para a idade, $77,2 \%$ e $80,3 \%$ encontravam-se na faixa de normalidade, $10,8 \%$ e $13,1 \%$ apresentavam risco de sobrepeso e $9,8 \%$ e $5 \%$

Tabela 1. Distribuição dos adolescentes segundo sexo e classificação do estado nutricional, Centro de Juventude da cidade de São Paulo, 2000.

\begin{tabular}{|c|c|c|c|c|c|c|}
\hline \multirow[b]{2}{*}{ Estado Nutricional } & \multicolumn{2}{|c|}{ Masculino $(n=92)$} & \multicolumn{2}{|c|}{ Feminino $(n=61)$} & \multicolumn{2}{|c|}{ Total $(n=153)$} \\
\hline & $n$ & $\%$ & $n$ & $\%$ & $n$ & $\%$ \\
\hline Baixo Peso & 2 & 2,2 & 1 & 1,6 & 3 & 2,0 \\
\hline Normalidade & 71 & 77,2 & 49 & 80,3 & 120 & 78,4 \\
\hline Risco de Sobrepeso & 10 & 10,8 & 8 & 13,1 & 18 & 11,8 \\
\hline Sobrepeso & 9 & 9,8 & 3 & 5,0 & 12 & 7,8 \\
\hline Total & 92 & 100,0 & 61 & 100,0 & 153 & 100,0 \\
\hline
\end{tabular}

Qui-quadrado=1,364; $p=0,714$. 
apresentou sobrepeso, respectivamente, sem diferenças estatisticamente significativas entre estado nutricional e sexo (Tabela 1).

Quanto à intensidade da atividade física, segundo sexo, observou-se diferença entre os adolescentes estudados ( $p>0,05$ estatisticamente significativa): os meninos revelaram predomínio da atividade intensa $(92,4 \%)$, e as meninas, de moderada $(45,9 \%)$ a intensa $(44,3 \%)$.

Analisando-se o consumo alimentar dos adolescentes de forma qualitativa, observou-se a presença diária das bebidas gaseificadas, balas, chicletes, salgadinhos empacotados, biscoitos recheados e doces em barra (doce de leite, pé de moleque, doce de amendoim) na maioria da população estudada (70\%). Por outro lado, as frutas e hortaliças aparecem apenas nas refeições realizadas no CJ.

Identificou-se consumo de energia e nutrientes superior para os indivíduos do sexo masculino (Tabela 2), detectando-se diferença estatisticamente significativa apenas para o cálcio $(p<0,05)$.

O consumo alimentar dos adolescentes mostrou-se insuficiente no aspecto energético e elevado tocante às proteínas. Não foi verificado consumo elevado de cálcio e ferro na população estudada. Comparativamente, os meninos mostraram maior consumo de colesterol do que as meninas (Tabela 3).

À quantidade consumida de proteínas ( $\mathrm{g} / \mathrm{kg} / \mathrm{dia})$ ultrapassou o ideal recomendado $(0,8-1,0 \mathrm{~g} / \mathrm{kg} / \mathrm{dia})$ tanto para os meninos $(1,74)$ quanto para as meninas $(1,57)$, superando o valor máximo permitido $(1,6 \mathrm{~g} / \mathrm{kg} / \mathrm{dia})$ no sexo masculino.

A distribuição média de macronutrientes das dietas apresentou densidade de $54,1 \%$ de carboidratos, $14,3 \%$ de proteínas e $31,3 \%$ de lipídios.

Tabela 2. Distribuição dos valores médios de energia e nutrientes consumidos pelos adolescentes segundo sexo e valores do teste de Kruskal-Wallis com respectivos valores de p em relação à média de consumo. Centro de Juventude da cidade de São Paulo, 2000.

\begin{tabular}{|c|c|c|c|c|c|}
\hline \multirow{2}{*}{ Nutrientes } & \multicolumn{3}{|c|}{ Média de consumo } & \multicolumn{2}{|c|}{ Teste estatístico } \\
\hline & Masculino $(n=92)$ & Feminino $(n=61)$ & Total $(n=153)$ & Krukal-Wallis & Valores de $p$ \\
\hline Energia (CAL) & 2026,1 & 1842,7 & 1953,0 & 2,909 & 0,900 \\
\hline Carboidratos (g) & 270,3 & 253,8 & 263,7 & 1,326 & 0,251 \\
\hline Proteínas (g) & 71,5 & 64,9 & 68,9 & 2,745 & 0,100 \\
\hline Lipídios (g) & 69,7 & 67,4 & 68,8 & 0,263 & 0,609 \\
\hline Cálcio (mg) & 580,0 & 421,0 & 516,7 & $11,984^{*}$ & $0,001^{*}$ \\
\hline Ferro (mg) & 10,1 & 9,4 & 9,8 & 1,007 & 0,317 \\
\hline Colesterol (mg) & 346,3 & 304,7 & 329,7 & 1,670 & 0,198 \\
\hline
\end{tabular}

${ }^{(*)}$ Valores estatisticamente significantes.

Tabela 3. Adequação do consumo alimentar dos adolescentes segundo sexo. Centro de Juventude da cidade de São Paulo, 2000.

\begin{tabular}{|c|c|c|c|c|c|c|}
\hline & \multicolumn{3}{|c|}{ Sexo masculino $(n=92)$} & \multicolumn{3}{|c|}{ Sexo feminino $(n=61)$} \\
\hline & Adequado & Insuficiente & Elevado & Adequado & Insuficiente & Elevado \\
\hline Energia & 21,7 & $76,1^{*}$ & 2,2 & 36,1 & 55,7 & 8,2 \\
\hline Proteínas & 14,2 & 7,6 & $77,2^{*}$ & 29,5 & 6,6 & $63,9^{*}$ \\
\hline Cálcio & 9,8 & $90,2^{*}$ & 0 & 1,6 & $98,4^{*}$ & 0 \\
\hline Ferro & 40,2 & 59,8 & 0 & 16,9 & $83,6^{*}$ & 0 \\
\hline Colesterol & 46,7 & 0 & 53,3 & 59,0 & 0 & 41,0 \\
\hline
\end{tabular}

${ }^{(*)}$ Valores estatisticamente significantes. 
Quanto ao valor de IQN para cálcio e ferro (Tabela 4), as dietas das meninas apresentaram resultados menos favoráveis do que aqueles verificados para os meninos, tendo sido registradas diferenças estatisticamente significativas $(p<0,05)$.

Com relação ao número de refeições realizadas, $94 \%$ dos adolescentes realizavam pelo menos três refeições diárias, sendo duas maiores (almoço e jantar) e uma menor. O café da manhã mostrou-se negligenciado por $14,8 \%$ das meninas e $9,8 \%$ dos meninos.
DISCUSSÃ O

A maioria dos adolescentes estudados apresentou nível nutricional dentro dos padrões de normalidade $(78,4 \%)$, sendo detectado risco de sobrepeso e sobrepeso instalado em 19,6\% deles. Esta proporção de sobrepeso mostra-se relativamente inferior à relatada em estudos realizados no Canadá, (O'Lougin et al., 1998; Hanley et al., 2000), Estados Unidos (McMurray et al., 2000) e Brasil (Nuzzo, 1998; Albano, 2000), os quais identificaram valores em torno de $30,0 \%$. É possível que o estímulo do CJ à prática de

Tabela 4. Distribuição dos adolescentes segundo sexo e Índice de Qualidade Nutricional - IQN para cálcio e ferro. Centro de Juventude da cidade de São Paulo, 2000.

\begin{tabular}{|c|c|c|c|c|c|c|}
\hline \multirow[b]{2}{*}{ Classificação de iqn } & \multicolumn{2}{|c|}{ Masculino $(n=92)$} & \multicolumn{2}{|c|}{ Feminino $(n=61)$} & \multicolumn{2}{|c|}{ Total $(n=153)$} \\
\hline & Cálcio* & Ferro** & Cálcio & Ferro & Cálcio & Ferro \\
\hline Pobre $(>0,5)$ & 23,9 & 2,8 & 59,0 & 14,8 & 42,4 & 7,2 \\
\hline Regular $(0,5-0,89)$ & 46,7 & 13,0 & 36,1 & 59,0 & 38,6 & 31,3 \\
\hline Adequado $(0,9-1,5)$ & 1,1 & 34,2 & 3,2 & 24,6 & 17,6 & 21,6 \\
\hline $\operatorname{Bom}(1,51-4,9)$ & 28,3 & 50,0 & 1,6 & 1,6 & 1,3 & 39,9 \\
\hline
\end{tabular}

${ }^{(*)}$ Qui-quadrado=29,231; $p>0,000 ;{ }^{(\star *)}$ Qui-quadrado $=59,802 ; p>0,000$.

Tabela 5. Média de consumo energético e de cálcio, por sexo, em estudos de consumo alimentar de adolescentes.

\begin{tabular}{|c|c|c|c|c|c|c|}
\hline \multirow{2}{*}{ Autores } & \multirow{2}{*}{$n$} & \multirow{2}{*}{ Idade (anos) } & \multicolumn{2}{|c|}{ Média de consumo energético (Cal) } & \multicolumn{2}{|c|}{ Média de consumo de cálcio (mg) } \\
\hline & & & Masculino & Feminino & Masculino & Feminino \\
\hline Albano et al. (2000) & 92 & $10-17$ & 2734 & 2197 & 819 & 579 \\
\hline Lerner et al. (2000) & 323 & $11-17$ & 2275 & 2009 & 628 & 565 \\
\hline Harel et al. (1998) & 1117 & $14,8 \pm 0,2$ & & & 681 & 536 \\
\hline \multirow[t]{3}{*}{ Nuzzo (1998) } & 200 & $10-12$ & 1921 a 2275 & 1750 a 2222 & & \\
\hline & & $12-15$ & 2278 a 2465 & 1615 a 2012 & & \\
\hline & & $15-18$ & 2414 a 2966 & 1615 a 2012 & & \\
\hline \multirow[t]{2}{*}{ Sichieri (1998) } & 540 & $12-14$ & 2885 & 2848 & 1061 & 1150 \\
\hline & & $15-18$ & 3025 & 2607 & 1114 & 1018 \\
\hline Ballew et al. (1997) & 162 & $12-19$ & 2226 & 2031 & 588 & 540 \\
\hline Lysen \& Walker (1997) & 138 & $10-14$ & & & 2744 & 2178 \\
\hline \multirow[t]{3}{*}{ Shatenstein \& Ghadirian (1996) } & 182 & $10-12$ & 1978 & 2005 & 1097 & 1043 \\
\hline & & $13-15$ & 2486 & 2033 & 1114 & 951 \\
\hline & & $15-18$ & 2909 & 1795 & 316 & 1006 \\
\hline Andersen et al. (1995) & 1564 & $18^{*}$ & $3776^{*}$ & $2366^{*}$ & $1628^{*}$ & $1078^{*}$ \\
\hline \multirow[t]{2}{*}{ Strain et al. (1994) } & 1015 & $12-13$ & 2629 & 2198 & & \\
\hline & & $13-15$ & 3130 & 2174 & & \\
\hline Roma-Giannikou et al. (1997) & 1936 & $10-14$ & & & 963 & 800 \\
\hline Fleming et al. (1994) & & $12-19$ & & & 1061 & 789 \\
\hline
\end{tabular}

${ }^{(*)}$ Em mediana 
exercícios físicos tenha constituído o diferencial observado.

Por outro lado, aquela proporção (19,6\%), embora inferior à encontrada na literatura, pode ser significativa se considerada a elevada prevalência de sobrepeso que vem sendo detectada entre crianças e adolescentes norte-americanas - National Health and Nutrition Examination Survey (NHANES) (Center..., 1997). Acresce-se a essa constatação o fato de existir associação de sobrepeso e obesidade com doenças crônicas não transmissíveis na idade adulta, como ressaltam Monteiro et al. (1995).

A elevada proporção $(92,4 \%)$ de adolescentes do sexo masculino que praticavam atividade física intensa pode ser justificada pelo incentivo do CJ ao treinamento esportivo competitivo, especialmente nas modalidades de futebol e capoeira, as preferidas entre os meninos. Adicionalmente, além das atividades no Centro, os jovens também freqüentam aula de educação física na escola onde estudam. Quanto aos dados referentes ao sexo feminino o predomínio da atividade moderada $(45,9 \%)$ entre as meninas vai ao encontro do relatado por Pimenta et al. (1999) e McMurray et al. (2000).

O fato de os adolescentes apresentarem consumo elevado de bebidas gaseificadas e alimentos ricos em açúcar e gordura, principalmente nas refeições intermediárias, assemelha-se às constatações de Andersen et al. (1995) e Anding et al. (1996). O baixo consumo de frutas, hortaliças e produtos lácteos é similar ao observado em outros estudos realizados com adolescentes (Gambardella, 1995; Gambardella et al., 1999; Lerner et al., 2000).

Conforme esperado, a média de consumo energético foi maior para o sexo masculino (2 026 Cal) do que para o feminino (1 842 (al); no entanto, o valor foi inferior ao registrado em outras pesquisas (Tabela 5).

O consumo insatisfatório de carboidratos e excessivo de lipídios e de proteínas é semelhante ao relatado na literatura (Strain et al., 1994; Andersen et al., 1995; Samuelson et al., 1996;
Shatenstein \& Ghadirian, 1996; Ballew et al., 1997; Hurson \& Corish., 1997; Roma-Giannikou et al., 1997; Nuzzo, 1998; Sichieri, 1998; Wang et al., 1998; Albano, 2000).

O déficit de consumo de cálcio $(515,4 \mathrm{mg})$ pode ser explicado, em grande parte, pela baixa ingestão de alimentos fontes deste nutriente (leites e derivados), informada pelos adolescentes, bem como pela substituição do leite por sucos industrializados no desjejum e refrigerantes nos lanches da manhã e tarde realizados fora do CJ. Soma-se a isso a proporção de indivíduos que informaram negligenciar o desjejum. Além disso, não foi referido consumo de queijo, talvez por ser alimento de elevado custo de comercialização para uma população de baixo nível socioeconômico. No entanto, vários trabalhos apresentam resultados semelhantes de inadequação de consumo de cálcio entre adolescentes, independentemente do nível socioeconômico, excetuando-se os valores registrados por Lysen \& Walker (1997) (Tabela 5).

Com relação ao ferro. Embora tenha sido elevada a proporção de meninas $(83,6 \%)$ que apresentaram baixo consumo de alimentos fontes deste mineral, não se encontrou consenso na literatura. Albano (2000) verificou média de ingestão de $13,73 \mathrm{mg}$ para meninos e $11,31 \mathrm{mg}$ para meninas. Sichieri (1998) defrontou-se com consumo médio para o sexo masculino de $17,7 \mathrm{mg}$, e, para o feminino de $16,1 \mathrm{mg}$. Andersen et al. (1995) constataram consumo mediano de $16 \mathrm{mg}$ para meninos e $11 \mathrm{mg}$ para meninas. Estudo realizado por Ortega et al. (1993) apontou ingestão de ferro para o sexo masculino de $16 \mathrm{mg}$ e para o sexo feminino de $11,3 \mathrm{mg}$.

A deficiência de ferro é um dos problemas nutricionais freqüentes entre os adolescentes e pode apresentar repercussões não só na saúde como também nas aptidões e rendimento escolar dos indivíduos (Ortega et al., 1993), podendo causar depressão do sistema imunológico, diminuição da síntese de neurotransmissores e mielina e anemia ferropriva (National Research..., 1989). 
Aproximadamente $50 \%$ da população estudada apresentou consumo elevado de colesterol. Sichieri (1998) também encontrou ingestão acima do recomendado, em estudo com 540 adolescentes de 10 a 18 anos, sendo a média para o sexo masculino, na faixa etária de 10 a 14 anos, de 403mg e para o sexo feminino, de $383 \mathrm{mg}$. Capper et al. (1990), em pesquisa realizada com 200 adolescentes norte-americanos com idade média de 15 anos, obtiveram média de consumo de colesterol de $468 \mathrm{mg} /$ dia para meninos $297 \mathrm{mg} / \mathrm{dia}$ para meninas.

A importância da redução na quantidade de gordura e colesterol está relacionada à alta prevalência de doenças cardíacas (National Research..., 1989). Entretanto, na literatura não têm sido encontrados muitos trabalhos com relato do consumo de colesterol no Brasil. Talvez o motivo seja a ausência de tabela de composição de alimentos que contenha a quantidade de colesterol dos alimentos, havendo a necessidade de os dados referentes a este nutriente são compilados de tabelas estrangeiras.

\section{CONCLUSÃO}

Os adolescentes estudados apresentaram adequado estado nutricional em sua maioria $(78,4 \%)$. No entanto, $19,6 \%$ encontraram-se na faixa de risco de sobrepeso e obesidade.

Verificou-se consumo alimentar elevado em relação à proteína e aos lipídios (colesterol) e insuficiente em cálcio e ferro. A insuficiência de cálcio foi observada na população em geral, mas houve maior prevalência entre as meninas $(98,4 \%)$, as quais também apresentaram maior proporção de qualidade da dieta "pobre" e "regular" em ferro (73,8\%).

Em relação às meninas, aumentar o consumo de energia e nutrientes poderia propiciar o alcance das necessidades, principalmente de energia, cálcio e ferro. Entretanto, elas ganhariam peso, passando de IMC normal para excesso de peso. Por outro lado, conforme muitos estudos vêm demonstrando, o consumo excessivo de alimentos ricos em gordura e açúcar, associado ao estilo de vida sedentário, propicia aumento da prevalência de obesidade na adolescência (Monteiro et al., 1995).

Nesse contexto, surge a dúvida quanto ao que seria melhor para os adolescentes estudados: elevar o consumo de energia e nutrientes no sentido de atingir o recomendado com prejuízo de ganho de peso? Além disso, como suprir as necessidades de cálcio e ferro sem aumentar as quantidades de proteína e gordura, principalmente colesterol, que já se apresentam elevadas?

Uma alternativa seria aumentar o consumo de produtos fontes de cálcio com reduzido conteúdo de gordura e de gêneros de origem vegetal fontes de ferro, associando-os com a ingestão de alimentos fontes de vitamina $C$.

Desse modo, denota-se a importância da promoção de educação nutricional entre jovens, com ênfase para a escolha apropriada dos alimentos, principalmente aqueles contidos nas refeições intermediárias.

\section{REFERÊ NCIAS B I B LIOGRÁFICAS}

ALBANO, R.B. Estado nutricional e consumo alimentar de adolescentes. São Paulo, 2000. 67p. Dissertação (Mestrado) - Faculdade de Saúde Pública, Universidade de São Paulo, 2000.

ANDERSEN, L.F., NES, M., SANDSTAD, B., BJORNEBOE, G.E., DREVON, C.A. Dietary intake among Norwegian adolescents. European Journal of Clinical Nutrition, London, v.49, n.8, p.555-564, 1995.

ANDING, J.D., KUBENA, K.S., McINTOSH, W.A., O'BRIEN, B. Blood lipids, cardiovascular fitness, obesity and blood pressure: the presence of potential coronary heart disease risk factors in adolescents. Journal of the American Dietetic Association, Chicago, v.96, n.3, p.238-242, 1996.

BALLEW, C., WHITE, L.L., STRAUSS, K.F., BENSON, L.J., MENDLEIN, J.M., MOKDAD, A.H. Intake of nutrients and food sources of nutrients among the Navajo: 
findings from the Navajo Health and Nutrition Survey. Journal of Nutrition, Bethesda, v.127, p.2085S-2093S, 1997. Supplement 10.

CAPPER, A.L., ELLISON, R.C., WITSCHI, J.C. Sources of fat, fatty acids, and cholesterol in the diets of adolescents. Journal of the American Dietetic Association, Chicago, v.90, n.10, p.1429-31, 1990.

CENTER FOR DISEASE CONTROL AND PREVENTION. Update: prevalence of overweight among children, adolescents and adults - United States, 1988 - 1994. Morbidity and Mortality Weekly Report, v.46, n.9, p.199-201, 1997.

DIETZ, W.H. Childhood weight affects adult morbidity and mortality. Journal of Nutrition, Bethesda, v.128, p.411S-414S, 1998. Supplement 2.

SÃO PAULO. Secretaria da Família e Bem-estar Social. Supervisão Geral de Planejamento e Controle. Política de Convênios da Secretaria Municipal da Família e Bem-estar Social. São Paulo, 1993. p.2-6, 15-18.

FLEMING, K.H., HEIMBACH, J.T. Consumption of calcium in the U.S.: food sources and intake levels. Journal of Nutrition, Bethesda, v.124, p.1426S-1430S, 1994. Supplement 8.

GAMBARDELLA, A.M.D. Adolescentes, estudantes de período noturno: como se alimentam e gastam suas energias. São Paulo, 1995. 81 p. Tese (Doutorado) - Faculdade de Saúde Pública, Universidade de São Paulo, 1995

GAMBARDELLA, A.M.D., FRUTUOSO, M.F.P., FERREIRA, C.F. Prática alimentar de adolescentes. Revista de Nutrição, Campinas, v.12, n.2, p.55-63, 1999.

GORDON, C.C., CHUMLED, W.C., ROCHE, A.F. Stature, recumbent length and weight. In: LOHMAN, T.G., ROCHE, A.F., MARTORELL, R. Anthropometric standardization reference manual. Champaign IL: Human Kinetics Books, 1988. 177p.

HANLEY, A.J., HARRIS, S.B., GITTELSOHN, J., WOLEVER, T.M., SAKSVIG, B., ZINMAN, B. Overweight among children and adolescents in a Native Canadian community: prevalence and associated factors. American Journal of Clinical Nutrition, Bethesda, v.71, n.3, p.693-700, 2000.

HAREL, Z., RIGGS, S., VAZ, R., WHITE, L., MENZIES, G. Adolescents and calcium: what they do and do not know and how much they consume. Journal of Adolescent Health, New York, v.22, n.3, p.225-228, 1998.

HURSON, M.M., CORISH, C. Evaluation of lifestyle, food consumption and nutrient intake patterns among Irish teenagers. Iris Journal of Medical Science, Dublin, v.166, n.4, p.225-30, 1997.

LERNER, B.R., LEI, D.L.M., CHAVES, S.P., FREIRE, R.D. O cálcio consumido por adolescentes de escolas públicas de Osasco, São Paulo. Revista de Nutrição, Campinas, v.13, n.1, p.57-63, 2000.

LYSEN, V.C., WALKER, R. Osteoporosis risks factors in eight grade students. Journal of School Health, Kent, v.67, n.8, p.317-321, 1997.

MCMURRAY, R.G., HARRELL, J.S., DENG, S., BRADLEY, C.B., COX, L.M., BANGDIWALA, S.I. The influence of physical activity, socioeconomic status and ethnicity on the weight status of adolescents. Obesity Research, v.8, n.2, p.130-139, 2000.

MAJEM, L.I.S., BARBA, L.R. Recordatório de 24 horas. In: MAJEN, L.I.S., BARTINA, J.A., VERDÚ, J.M. Nutrición y salud pública: métodos, base científicas y aplicaciones. Barcelona : Masson, 1995. p.113-119.

MONTEIRO, C.A., MONDINI, L., SOUZA, A.L.M., POPKIN, B.M. Da desnutrição para obesidade: a transição nutricional no Brasil. In: MONTEIRO, C.A. Velhos e novos males da Saúde no Brasil: a evolução do país e suas doenças. São Paulo : Hucitec, 1995.

NATIONAL RESEARCH COUNCIL (USA). Recommended dietary allowances. 10.ed. Washington DC : National Academy Press, 1989. 284p.

NUZZO, L. Avaliação do estado nutricional de adolescentes de uma escola privada de ensino. São Paulo, 1998. 69p. Dissertação (Mestrado) - Faculdade de Saúde Pública, Universidade de São Paulo, 1998.

O'LOUGHLIN, J., PARADIS, G., RENAUD, L., MESHEFEDJIAN, G., GRAY-DONALD, K. Prevalence and correlates of overweight among elementary schoolchildren in multiethnic, low income, inner-city, neighborhoods in Montreal, Canada. Annals of Epidemiology, New York, v.8, n.7, p.422-432, 1998. 
ORGANIZAÇÃO MUNDIAL DA SAÚDE. Reunião Mista. Necessidades de energia e proteína. São Paulo : Roca, 1998. 225p. (OMS - Série de Relatos Técnicos, 724).

ORGANIZACIÓN MUNDIAL DE LA SALUD. La salud de los jóvenes: un reto y una esperanza. Ginebra, 1995. 120p.

ORTEGA, A.R.M., GONZÁLES-FERNANDÉZ, M., PAZ, L., ANDRÉS, P., JIMÉNEZ, L.M., JIMÉNEZ, M.J. Influencia del status en hierro en la atención y rendimlento intelectual de un colectivo de adolescentes españoles. Archivos Latinoamericanos de Nutrición, Guatemala. v.43, n.1, p.6-11, 1993.

PIMENTA, C.D., GAMBARDELLA, A.M.D., CARDOSO, M.R.A., BISMARCK-NASR, E.M. Relação entre a taxa de gordura corporal e assistir televisão em adolescentes de diferentes níveis socioeconômicos. Revista Brasileira de Ciência e Movimento, São Paulo, (edição especial), 1999. p.154.

REINSTEM, C.S.B. Diet Win Clínico. Software Avaliação Nutricional. Versão 2.1.8. Rio Grande do Sul, 1998.

ROMA-GIANNIKOU, E., ADAMIDIS, D., GIANNIOU, M., NIKOLARA, R., MATSANIOTIS, N. Nutritional survey in Greek children: nutrient intake. European Journal of Clinical Nutrition, London, v.51, n.5, p.273-285, 1997.

SAMUELSON, G., BRATTEBY, L.E., ENGHARDT, H., HEDGREN, M. Food habits and energy and nutrient intake in Swedish adolescents approaching the year 2000. Acta Paediatrica, Stockholm, v.415, p.1-19, 1996. Supplement.

SICHIERI, R. Epidemiologia da obesidade. Rio de Janeiro : UERJ, 1998. 139p.
STATISTICAL Package for the Social Sciences for Windows Student Version. Release 7.5. Marketing Department. Chicago, 1996.

SHATENSTEIN, B., GHADIRIAN, P. Nutrient patterns and nutritional adequacy among French-Canadian children in Montreal. Journal of the American College of Nutrition, Quebec, v.15, n.32, p.264-72, 1996.

STRAIN, J.J., ROBSON, P.J., LIVINGSTONE, M.B., PRIMROSE, E.D., SAVAGE, J.M., CRAN, G.W., BOREHAM, C.A. Estimates of food and macronutrient intake in a random sample of Northern Ireland adolescents. Journal of Nutrition, Bethesda, v.72, n.3, p.343-352, 1994.

WANG, Y., POPKIN, B., ZHAI, F. The nutritional status and dietary pattern of Chinese adolescents, 1991 and 1993. European Journal of Clinical Nutrition, London, v.52, n.12, p.908-916, 1998.

WITTWER, A.J., SORENSON, A.W., WYSE, B.W., HANSEN, R.G. Nutrient density: evaluation of nutritional attributes of foods. Journal of Nutritional Education, California, v.9, p.26-30, 1977.

WORLD HEALTH ORGANIZATION. Physical status: the use and interpretation of anthropometry. Geneva, 1995. p.4-36 (Technical Report Series, 854).

WORLD HEALTH ORGANIZATION. Obesity: preventing and managing the global epidemic. Geneva, 1997 (Report of a WHO Consultation on Obesity).

ZABOTTO, C.B., VEANNA, R.P.T., GIL, M.F. Registro fotográfico para inquéritos dietéticos: utensílios e porções. Goiânia : Nepa-Unicamp; 1996.

Recebido para publicação em 20 de fevereiro de 2001 e aceito em 7 de fevereiro de 2002. 